\title{
A REFINEMENT OF HADAMARD'S INEQUALITY FOR ISOTONIC LINEAR FUNCTIONALS
}

\author{
SEVER SILVESTRU DRAGOMIR
}

A refinement of Hadamard's inequality for isotonic linear functionals and some applications to norm and discrete inequalities are given.

\section{Introduction}

Let $f: I \subseteq \mathbb{R} \rightarrow \mathbb{R}$ be a convex function. The following double inequality

$$
f\left(\frac{x+y}{2}\right) \leq \frac{1}{y-x} \int_{x}^{y} f(t) d t \leq \frac{f(x)+f(y)}{2}
$$

where $x, y \in I, x<y$, is known in literature as Hadamard's inequality (see [6], [9] or [5]). For some recent results in connection with this famous integral inequality we refer to [2-5] and [9-11] where further applications are given.

In this paper we will give an analogous of this fact for isotonic linear functionals (compare with [8]). Some natural applications are also pointed out.

As in [1], let $E$ be a nonempty set and let $L$ be a linear class of real valued functions $g: E \rightarrow \mathbb{R}$ having the properties:

$$
\begin{aligned}
& L 1: f, g \in L \text { imply }(a f+b g) \in L \text { for all } a, b \in \mathbb{R} ; \\
& L 2: \mathbb{1} \in L, \text { that is, if } f(t)=1(t \in E) \text { then } f \in L .
\end{aligned}
$$

We also consider isotonic linear functionals $A: L \rightarrow \mathbb{R}$. That is, we suppose:

$$
\begin{aligned}
& A 1: A(a f+b g)=a A(f)+b A(g) \text { for all } f, g \in L \text { and } a, b \in \mathbb{R} ; \\
& A 2: f \in L, f(t) \geq 0 \text { on } E \text { implies } A(f) \geq 0 .
\end{aligned}
$$


We note that common examples of such isotonic linear functionals $A$ are given by $A(g):=\int_{E} g d \mu$ or $A(g)=\sum_{k \in E} p_{k} g_{k}$ where $\mu$ is a positive measure on $E$ in the first case and $E$ is a subset of natural number $\mathbb{N}$ in the second case with $p_{k} \geq 0$ for $k \in E$.

We also will use Jensen's inequality (see e.g. [1]):

Theorem 1.1. Let $L$ satisfy properties $L 1, L 2$ on a nonempty set $E$ and suppose $\phi$ is a convex function on an interval $I \subseteq \mathbb{R}$. If $A$ is any isotonic linear functional with $A(\mathbb{1})=1$, then for all $g \in L$ so that $\phi(g) \in L$, we have $A(g) \in I$ and

$$
\phi(A(g)) \leq A(\phi(g))
$$

\section{The Main Result}

We will start with the following simple lemma.

Lemma 2.1. Let $X$ be a real linear space and $C$ be its convex subset. If $f: C \rightarrow \mathbb{R}$ is convex on $C$, then for all $x, y$ in $C$ the mapping $g_{x, y}:[0,1] \rightarrow \mathbb{R}$ given by

$$
g_{x, y}(t):=1 / 2[f(t x+(1-t) y)+f((1-t) x+t y)]
$$

is also convex on $[0,1]$. In addition, we have the inequality:

$$
f\left(\frac{x+y}{2}\right) \leq g_{x, y}(t) \leq \frac{f(x)+f(y)}{2}
$$

for all $x, y$ in $C$ and $t \in[0,1]$.

Proof. Suppose $x, y \in C$ and let $t_{1}, t_{2} \in[0,1]$ and $\alpha, \beta \geq 0$ with $\alpha+\beta=1$. Then

$$
\begin{aligned}
& g_{x, y}\left(\alpha t_{1}+\beta t_{2}\right) \\
= & \frac{1}{2}\left(f\left[\left(\alpha t_{1}+\beta t_{2}\right) x+\left(1-\alpha t_{1}-\beta t_{2}\right) y\right]+f\left[\left(1-\alpha t_{1}-\beta t_{2}\right) x+\left(\alpha t_{1}+\beta t_{2}\right) y\right]\right) \\
= & \frac{1}{2}\left(f \left[\alpha\left(t_{1} x+\left(1-t_{1}\right) y\right)+\beta\left(t_{2} x+\left(1-t_{2}\right) y\right]\right.\right. \\
& \left.+f\left[\alpha\left(\left(1-t_{1}\right) x+t_{1} y\right)+\beta\left(\left(1-t_{2}\right) x+t_{2} y\right)\right]\right)
\end{aligned}
$$




$$
\begin{aligned}
\leq & \frac{1}{2}\left(\alpha f\left[t_{1} x+\left(1-t_{1}\right) y\right]+\beta f\left[t_{2} x+\left(1-t_{2}\right) y\right]\right. \\
& \left.\left.+\alpha f\left[\left(1-t_{1}\right) x+t_{1} y\right]+\beta f\left[\left(1-t_{2}\right) x+t_{2} y\right)\right]\right) \\
= & \alpha g_{x, y}\left(t_{1}\right)+\beta g_{x, y}\left(t_{2}\right)
\end{aligned}
$$

which shows that $g_{x, y}$ is convex on $[0,1]$.

By the convexity of $f$ we can state:

$$
g_{x, y}(t) \geq f\left[\frac{1}{2}(t x+(1-t) y+(1-t) x+t y)\right]=f\left(\frac{x+y}{2}\right)
$$

and also

$$
g_{x, y}(t) \leq \frac{1}{2}[t f(x)+(1-t) f(y)+(1-t) f(x)+t f(y)]=\frac{f(x)+f(y)}{2}
$$

for all $t$ in $[0,1]$, which completes the proof.

Remark 2.2. By the inequality (2.1) we can state:

$$
\sup _{t \in[0,1]} g_{x, y}(t)=\frac{f(x)+f(y)}{2} \text { and } \inf _{t \in[0,1]} g_{x, y}(t)=f\left(\frac{x+y}{2}\right)
$$

for all $x, y$ in $C$.

Now, we can give our main result.

Theorem 2.3. Let $f: C \subseteq X \rightarrow \mathbb{R}$ be a convex function on convex set $C$, $L$ and $A$ satisfy conditions $L 1, L 2$ and $A 1, A 2$ and $h: E \rightarrow \mathbb{R}, 0 \leq h(t) \leq 1$ $(t \in E), h \in L$ is so that $f(h x+(1-h) y), f((1-h) x+h y)$ belong to $L$ for $x, y$ fixed in $C$. If $A(\mathbb{1})=1$, then we have the inequality:

$$
\begin{aligned}
f\left(\frac{x+y}{2}\right) & \leq \frac{1}{2}[f(A(h) x+(1-A(h)) y)+f((1-A(h)) x+A(h) y)] \\
& \leq \frac{1}{2}(A[f(h x+(1-h) y)]+A[f((1-h) x+h y)]) \\
& \leq \frac{f(x)+f(y)}{2} .
\end{aligned}
$$

Proof. Let consider the mapping $g_{x, y}:[0,1] \rightarrow \mathbb{R}$ given above. Then by the above lemma we know that $g_{x, y}$ is convex on $[0,1]$. Applying Jessen's inequality for the mapping $g_{x, y}$ we get

$$
g_{x, y}(A(h)) \leq A\left(g_{x, y}(h)\right) .
$$


But

$$
g_{x, y}(A(h))=\frac{1}{2}[f(A(h) x+(1-A(h)) y)+f((1-A(h)) x+A(h) y)]
$$

and

$$
A\left(g_{x, y}(h)\right)=\frac{1}{2}(A[f(h x+(1-h) y]+A[f((1-h) x+h y)])
$$

and the second inequality in (2.2) is proved.

To prove the first inequality in (2.2) we observe that, by (2.1), we can write

$$
f\left(\frac{x+y}{2}\right) \leq g_{x, y}(A(h))
$$

which is ecxactly the desired statement.

Finally, we observe that, by the convexity of $f$, we get

$$
\frac{1}{2}[f(h x+(1-h) y)+f((1-h) x+h y)] \leq \frac{f(x)+f(y)}{2} \text { on } \mathbb{E} \text {. }
$$

Applying to this inequality the functional $A$ and since $A(\mathbb{1})=1$, we obtain the last part of (2.2).

Remark 2.4. If we chose: $A=\int_{0}^{1}, E=[0,1], h(t)=t, C=[x, y] \subset \mathbb{R}$ and since a simple calculation shows that

$$
\int_{0}^{1} f(t x+(1-t) y) d t=\int_{0}^{1} f((1-t) x+t y) d t=\frac{1}{y-x} \int_{x}^{y} f(t) d t
$$

we recapture, by (2.2) the inequality (1.1) of Hadamard.

\section{Applications}

1. Let $h:[0,1] \rightarrow[0,1]$ be a Riemann integrable function on $[0,1]$ and $p \geq 1$. Then for all $x, y$ vectors in normed space $(X ;\|\cdot\|)$ we have the inequality:

$$
\begin{aligned}
\left\|\frac{x+y}{2}\right\|^{p} \leq & \frac{1}{2}\left[\left\|\left(1-\int_{0}^{1} h(t) d t\right) x+\left(\int_{0}^{1} h(t) d t\right) y\right\|^{p}\right. \\
& \left.\quad+\left\|\left(\int_{0}^{1} h(t) d t\right) x+\left(1-\int_{0}^{1} h(t) d t\right) y\right\|^{p}\right] \\
\leq & \frac{1}{2}\left[\int_{0}^{1}\|h(t) x+(1-h(t)) y\|^{p} d t+\int_{0}^{1}\|(1-h(t)) x+h(t) y\|^{p} d t\right] \\
\leq & \frac{\|x\|^{p}+\|y\|^{p}}{2}
\end{aligned}
$$


If we chose $h(t)=t$, we obtain

$$
\left\|\frac{x+y}{2}\right\|^{p} \leq \int_{0}^{1}\|t x+(1-t) y\|^{p} d t \leq \frac{\|x\|^{p}+\|y\|^{p}}{2}
$$

for all $x, y$ in $X$.

The inequality (3.1) follows by Theorem 2.3 for the functional $A=\int_{0}^{1}$.

2. Let $f: C \subseteq X \rightarrow \mathbb{R}$ be a convex function on convex set $C$ of a linear space $X, t_{i} \in[0,1], i=1, \ldots, n$. Then we have the inequality:

$$
\begin{aligned}
f\left(\frac{x+y}{2}\right) & \leq \frac{1}{2}\left(f\left[\frac{1}{n} \sum_{i=1}^{n} t_{i} x+\frac{1}{n} \sum_{i=1}^{n}\left(1-t_{i}\right) y\right]+f\left[\frac{1}{n} \sum_{i=1}^{n}\left(1-t_{i}\right) x+\frac{1}{n} \sum_{i=1}^{n} t_{i} y\right]\right) \\
& \leq \frac{1}{2 n}\left[\sum_{i=1}^{n} f\left(t_{i} x+\left(1-t_{i}\right) y\right)+\sum_{i=1}^{n} f\left(\left(1-t_{i}\right) x+t_{i} y\right)\right] \\
& \leq \frac{f(x)+f(y)}{2}
\end{aligned}
$$

If we put $t_{i}=\sin ^{2} \alpha_{i}, \alpha_{i} \in \mathbb{R}, i=1, \ldots, n$, then we obtain

$$
\begin{aligned}
f\left(\frac{x+y}{2}\right) \leq & \frac{1}{2}\left(f\left[\left(\frac{1}{n} \sum_{i=1}^{n} \sin ^{2} \alpha_{i}\right) x+\left(\frac{1}{n} \sum_{i=1}^{n} \cos ^{2} \alpha_{i}\right) y\right]\right. \\
& \left.+f\left[\left(\frac{1}{n} \sum_{i=1}^{n} \cos ^{2} \alpha_{i}\right) x+\left(\frac{1}{n} \sum_{i=1}^{n} \sin ^{2} \alpha_{i}\right) y\right]\right) \\
\leq & \frac{1}{2 n} \sum_{i=1}^{n}\left(f\left[\left(\sin ^{2} \alpha_{i}\right) x+\left(\cos ^{2} \alpha_{i}\right) y\right]+f\left[\left(\cos ^{2} \alpha_{i}\right) x+\left(\sin ^{2} \alpha_{i}\right) y\right]\right) \\
\leq & \frac{f(x)+f(y)}{2}
\end{aligned}
$$

The inequaltity (3.3) follows by (2.2) for $A=1 / n \sum_{i=1}^{n}, h(i)=t_{i} \in[0,1]$.

By the use of the inequality (3.3), we can obtain a refinement of the arithmetic mean-geometric mean inequality

$$
\frac{x+y}{2} \geq \sqrt{x y} \text { where } x, y \geq 0 \text {. }
$$


Indeed, chosing $f(x):=-\ln x, x>0$, we obtain

$$
\begin{aligned}
\frac{x+y}{2} \geq & {\left[\left(\frac{1}{n} \sum_{i=1}^{n} t_{i}\right) x\right.} \\
& \left.+\frac{1}{n} \sum_{i=1}^{n}\left(1-t_{i}\right) y\right]^{1 / 2}\left[\frac{1}{n} \sum_{i=1}^{n}\left(1-t_{i}\right) x+\left(\frac{1}{n} \sum_{i=1}^{n} t_{i}\right) y\right]^{\frac{1}{2}} \\
\geq & \prod_{i=1}^{n}\left(\left[t_{i} x+\left(1-t_{i}\right) y\right]^{1 / 2}\left[\left(1-t_{i}\right) x+t_{i} y\right]^{1 / 2}\right)^{1 / n} \geq \sqrt{x y} .
\end{aligned}
$$

The equality holds in the previous inequalities simultaneously iff $x=y$.

\section{References}

[1] P. R. Beesack and J. E. Pečarić, "On Jessen's inequality for convex functions", J. Math. Anal. Appl., 110 (1985), 536-552.

[2] S. S. Dragomir, J. E. Pečarić and J. Sándor, "A note on the Jensen-Hadamard inequalities", Anal. Num. Theor. Approx., 19 (1990), 29-34.

[3] S. S. Dragomir, "Some refinements of Hadamard's inequalities", G. M. Metod. (Bucharest), 11 (1990), 189-191.

[4] S. S. Dragomir, "Two refinements of Hadamard's inequalities", Coll. of Sci. Pap. of the Fac. Science, Kragujevać, 11 (1990), 23-26.

[5] S. S. Dragomir, "A mapping in connection to Hadamard's inequalities", Anz. Österr. Akad. Wiss. Math.-natur. Klasse, 128 (1991), 17-20.

[6] J. Hadamard, "Etude sur les propriétés des fonctions entières et en particulier d'une fonction considérée par Riemann", J. Math. Pure Appl. 58 (1893), 171-215.

[7] J. L. W. V. Jensen, "Sur les fonctions convexes et les inégalités entres les valeurs moyenes", Actas Math. 30 (1906), 175-193.

[8] A. Lupas, "A generalization of Hadamard's inequalities for convex functions", Univ. Beograd Publ. Elektr. Fak. Ser. Mat. Fiz., No. 544-No. 576 (1976), 115-121.

[9] D. S. Mitrinović and I. B. Lacković, "Hermite and convexity", Aequat. Math., 28 (1985), 225-232.

[10] J. Sándor, "Some integral inequalities", El. Math. 43 (1988), 177-180.

[11] J. Sándor, "An application of the Jensen-Hadamard inequality", Nieuw Arch. Wiskunde (to appear).

Department of Mathematics, University of Timişoara, B-dul V. Pârvan 4, R-1900 Timişoara, România. 